\title{
Erratum: Enhancing Entropy and Enthalpy Fluctuations to Drive Crystallization in Atomistic Simulations [Phys. Rev. Lett. 119, 015701 (2017)]
}

Pablo M. Piaggi, Omar Valsson, and Michele Parrinello

(Received 9 September 2020; published 5 October 2020)

DOI: 10.1103/PhysRevLett.125.159902

We recently discovered an error in the calculation of the virial in our simulations. This error stemmed from a problem in the interface between the molecular dynamics engine LAMMPS and the enhanced sampling plugin PLUMED. The interface was available as a patch included in PLUMED at the time. When the potential energy is used as a collective variable, the forces and virial of the potential energy have to be scaled by an appropriate factor. In our simulations the forces were scaled appropriately but the virial was not. As a result of this issue the constant pressure simulations described in the Letter were effectively performed with an incorrect pressure estimator. Thus, the free energies and melting temperatures reported in the paper are also incorrect. Since the publication of our paper, a built-in PLUMED interface has been implemented in LAMMPS and the problem that we experienced has been solved. In this Erratum we report the results of new calculations. The findings and conclusions of the paper are not altered, but as we shall see, numerical results do differ.

We performed metadynamics simulations of $\mathrm{Na}$ and $\mathrm{Al}$ using the same parameters described in the Letter. From these simulations we calculated the difference in free energy between the liquid and the solid $\Delta G_{S \rightarrow L}$ as a function of simulation time. Figure 3 shows $\Delta G_{S \rightarrow L}$ as a function of temperature and is equivalent to Fig. 2 in the Letter. Melting temperatures for $\mathrm{Na}$ and $\mathrm{Al}$ were previously reported to be 340 and $800 \mathrm{~K}$, while the new calculations yield $367 \pm 7$ and $903 \pm 14 \mathrm{~K}$, respectively. These results are in much better agreement with the results obtained from coexistence simulations, namely, $366 \mathrm{~K}$ for $\mathrm{Na}$ and $926 \mathrm{~K}$ for Al. We note that finite size effects should increase the melting temperature. Therefore our estimation of the melting temperature of $\mathrm{Al}(903 \mathrm{~K})$, lower than the result from coexistence simulations ( $926 \mathrm{~K}$ ), has an error source different from finite size effects. We suggest that the sources of error are the formation of crystals with small strains and/or defects, and the statistical uncertainty of $\Delta G_{S \rightarrow L}$. We also recalculated the difference in entropy between the solid and the liquid at the melting temperature from the slope of the free energy. The results are 6.5 and $12.1 \mathrm{~J} /(\mathrm{mol} \mathrm{K})$ per atom for $\mathrm{Na}$ and $\mathrm{Al}$, respectively.

The new marginal probability distributions do not differ significantly from the ones reported in Fig. 1 of the Letter: Fig. 1(a) would correspond to $\mathrm{Na}$ at $\sim 375 \mathrm{~K}$ and Fig. 1(b) would correspond to $\mathrm{Al}$ at $\sim 900 \mathrm{~K}$, at variance with the reported temperatures 350 and $800 \mathrm{~K}$, respectively.
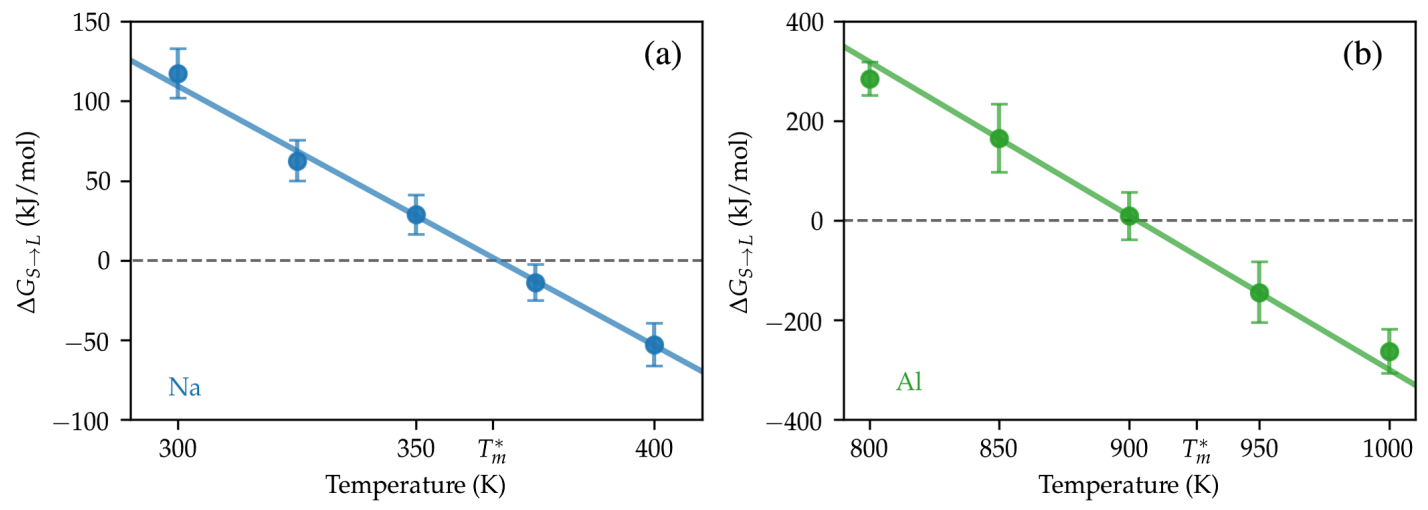

FIG. 1. Differences in free energy between the liquid and solid phases $\left(\Delta G_{S \rightarrow L}\right)$ as a function of temperature. Subplots (a) and (b) correspond to $\mathrm{Na}$ and $\mathrm{Al}$, respectively. The melting temperatures from coexistence simulations are marked in the abscissa axis with $T_{m}^{*}$. 